\title{
Aprovechamiento de ñandú (Rhea americana) en la prehistoria del sudeste uruguayo
}

\author{
FEDERICA MORENO \\ Departamento de Biodiversidad y Genética, \\ Instituto de Investigaciones Biológicas Clemente Estable-MEC, Uruguay
}

(Recibido 9 junio 2017; Revisado 3 noviembre 2017; Aceptado 5 diciembre 2017)

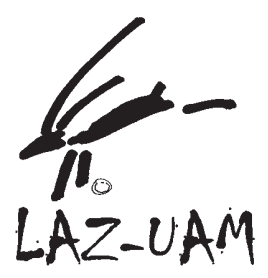

RESUMEN: El sitio Ch2D01 es un conjunto de dos montículos y varios microrelieves ubicado en el sudeste uruguayo, cuya ocupación data del Holoceno tardío. En este trabajo se analizan los restos óseos de ñandú (Rhea americana) recuperados en uno de los montículos (excavación IA). En este conjunto zooarqueológico, el ñandú representa únicamente el 0,5\% de los restos determinados a nivel de especie y género y el $0,1 \%$ del total. Los 20 restos identificados representan un $\mathrm{NMI}=5$ y corresponden en todos los casos a elementos de los miembros posteriores (tibiotarso, tarsometatarso y falanges). A pesar de esto, constituye un caso excepcional para la región donde los restos óseos de ñandú están ausentes en la mayoría de los sitios arqueológicos. Esta situación es similar a la descrita en diversos trabajos de sitios ubicados en la Pampa y Patagonia argentinas: baja presencia de ñandú y sobrerepresentación de elementos de las extremidades traseras. Los resultados son discutidos teniendo en cuenta la anatomía económica del animal, su densidad mineral ósea y las propuestas realizadas para los yacimientos argentinos. La representatividad anatómica es más coherente con un sitio de consumo de carne, médula y productos no comestibles que con un sitio de captura o de consumo de grasa. No se encontró correlación entre los elementos recuperados y la densidad mineral ósea, aunque lo reducido de la muestra no permite descartar del todo problemas de equifinalidad

PALABRAS CLAVE: RHEA AMERICANA, SUDESTE URUGUAYO, HOLOCENO TARDÍO, DIVERSIDAD ANATÓMICA

\begin{abstract}
The Ch2D01 site is a set of two earth mounds plus several minor earth structures located in the Uruguayan southeast, whose occupation dates from the late Holocene. In this work, the bone remains of rhea (Rhea americana) recovered in one mound (excavation IA) are analyzed. In this zooarchaeological assemblage, rhea represents only $0.5 \%$ of the remains determined at species and genus level and $0.1 \%$ of the total sample. The 20 remains identified represent an MNI $=5$ and correspond in all cases to elements of the hind limbs (tibiotarsus, tarsometatarsus and phalanges). This is an exceptional finding for the region where bones of rhea are absent from most archaeological sites. This situation is similar to that described in several sites located in the argentinean Pampa and Patagonia: low archaeological presence of rhea and dominant of hind limb elements. The results are discussed taking into account the economic anatomy of the animal, its mineral bone density and the explanations proposed for the Argentinian deposits. The anatomical representativeness is more consistent with a site of meat consumption, marrow and inedible products than with a kill site of capture or one of fat consumption. No correlation was found between the recovered elements and the bone mineral density, although the reduced sample does not allow to completely rule out problems of equifinality.
\end{abstract}




\section{INTRODUCCIÓN}

Los grupos humanos que ocuparon el sudeste uruguayo desde el Holoceno medio hasta el siglo XVI se caracterizaron por la construcción de miles de montículos de tierra, manejo de vegetales, control territorial, progresivo sedentarismo en aldeas, surgimiento de grupos diferenciados de individuos y espacios formales de inhumación (López Mazz, 2001; Cabrera, 2005; Gianotti, 2005; Bracco, 2006; Iriarte, 2006). Es una región ecotonal, y en ella convergen ambientes de costa atlántica, humedales de agua dulce, praderas, sierra (máx. $500 \mathrm{msnm}$ ), monte ribereño, palmares y lagunas costeras. Esta conjunción de ambientes resulta en una alta biodiversidad florística y faunística (PROBIDES, 1999). Climáticamente se ubica en una zona templada, con influencia de la región subtropical (Bracco et al., 2008). Los conjuntos zooarqueológicos regionales muestran la explotación de especies de pradera y humedal, entre las que destacan: cérvidos (Ozotoceros bezoarticus, Blastocerus dichotomus, Mazama gouazoubira), roedores medianos a grandes (Cavia sp., Myocastor coypus, Hydrochoerus hydrochaeris), armadillos (Dasypus sp., Eufractus sexcintus) y varias especies de peces (Silurifomes; Pogonias cromis; Micropogonias furnieri) (Pintos, 2000; Capdepont \& Pintos, 2006; Iriarte, 2006; Moreno, 2016; Moreno et al., 2016). En general, el ñandú (Rhea americana) muestra una presencia muy marginal, y son más frecuentes los fragmentos de cáscara de huevo que los restos óseos (Pintos, 1995; Iriarte, 2003; Brum, 2009). En esta región el único resto óseo de ñandú reportado hasta el momento se recuperó en la excavación del montículo B del sitio Ch2D01 (Bañado de San Miguel, Rocha) y se trata de un fragmento distal de tarsometatarso con marcas de carnicería (Pintos \& Gianotti, 1995). La baja presencia arqueológica de rheidos también se reporta en sitios de Pampa y Patagonia (Argentina), junto con una fuerte tendencia a la representación de elementos correspondientes a los miembros posteriores (Belardi, 1999; Fernández, 2000; Cruz \& Elkin, 2003; Cruz, 2005; Acosta \& Loponte, 2010; Frontini \& Picasso, 2010; Frontini, 2013).

En este trabajo se analizan los restos óseos de ñandú recuperados en el montículo A del sitio Ch2D01 (excavación IA). Para determinar si las frecuencias anatómicas presentes responden a decisiones económicas u otros procesos, los resultados son discutidos teniendo en cuenta la anatomía económica del animal (Giardina, 2006), su densi- dad mineral ósea (Cruz \& Elkin, 2003) y las explicaciones propuestas para los yacimientos argentinos (Fernández, 2000).

\section{EL ÑANDÚ (RHEA AMERICANA)}

La familia Rheidae (del grupo de las ratites) está conformada por dos especies: ñandú (Rhea americana) y ñandú petiso o choique (Rhea pennata). Su distribución geográfica se extiende desde el norte de Brasil hasta el norte de la Patagonia argentina. El ñandú es un ave corredora, posee alas reducidas, rectrices atrofiadas y quilla esternal muy poco desarrollada. En adultos, el plumaje es gris, con cobertoras blancas en las patas y dorso del cuerpo y zonas más oscuras en la corona, base del cuello, y pecho. Los tarsos son largos y no emplumados. Los machos adultos alcanzan hasta $150 \mathrm{~cm}$ de altura y $40 \mathrm{~kg}$ de peso y las hembras raramente superan los $130 \mathrm{~cm}$ y los $25-30 \mathrm{~kg}$. El ñandú tiene un comportamiento gregario, y puede formar bandadas de hasta 50 individuos, su alimentación es omnívora y ocupa campos abiertos y zonas poco arboladas (Bazzano et al., 2002; INAC, 2003; Reboreda \& Fernández, 2005). La madurez reproductiva se alcanza a los 2 años y la época de puesta de huevos va de setiembre a diciembre. Anida en el piso, y es el macho el que construye el nido, incuba los huevos y cría a los pichones. Una nidada puede contar con entre 20 a 50 huevos, la incubación empieza el día de la puesta del primer huevo, y se extiende por 36-37 días. La eclosión de los huevos es sincrónica, independientemente de las diferencias en los momentos de la puesta (Reboreda \& Fernández, 2005).

La anatomía económica de los rheidos muestra que los mayores valores de rendimiento de carne se encuentran en la cintura pélvica y el miembro posterior (fémur y tibiotarso), los de grasa en la cintura pélvica y el esternón y los de médula en el tibiotarso, fémur y tarsometatarso (Giardina, 2006). Por otro lado, estas aves proveen de una serie de productos no alimenticios como plumas, tendones, piel y huesos.

Estudios de densidad mineral ósea realizados sobre Rhea pennata muestran que los valores más altos de densidad estructural se ubican en la diáfisis de los huesos largos de la extremidad posterior (Cruz \& Elkin, 2003). Giardina (2006) encuentra en los rheidos cierto grado de correlación entre las partes de mayor rendimiento y las de mayor densi- 
dad mineral, lo que alerta sobre la probable existencia de problemas de equifinalidad en los conjuntos zooarqueológicos. De acuerdo a los índices de rendimiento, es esperable la presencia arqueológica de la cintura pélvica y el fémur en los sitios de consumo dado sus altos valores de utilidad cárnica. En los sitios de captura es esperable que se encuentren las vértebras cervicales, el miembro anterior y las falanges del miembro posterior. Si se aprovecha la grasa, se esperaría encontrar en los sitios de consumo esternón, cintura pélvica y escápula-coracoides. En el caso de aprovechamiento de médula, tendones y piel la expectativa es hacia miembros posteriores, incluyendo las falanges (Giardina, 2006).

\section{MATERIALES Y MÉTODOS}

El sitio Ch2D01 es un conjunto de dos montículos de tierra (denominados A y B) localizado en el Bañado de San Miguel, Departamento de Rocha (Figura 1). El montículo A fue excavado entre los años 1988 y 1992. Se realizaron dos excavaciones, en la parte central y en la ladera del montículo, denominadas IA y IC respectivamente (Curbelo et al.,

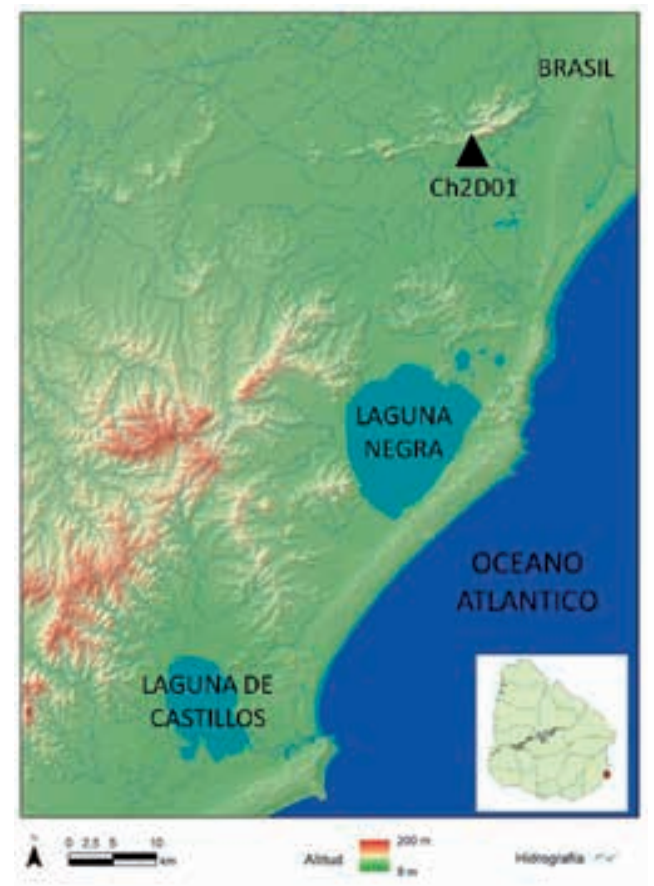

FIGURA 1

Localización geográfica del sitio Ch2D01.
1990). La excavación IA contó con una superficie total de $25 \mathrm{~m}^{2}$, que se completaron en dos etapas con una de $9 \mathrm{~m}^{2}(3 \times 3)$ y una ampliación periférica a ésta de $16 \mathrm{~m}^{2}$. Se recuperaron 20 conjuntos óseos humanos que, junto con elementos aislados, representan un número mínimo de 21 individuos de ambos sexos y edades comprendidas entre 2-3 y 55 años (Femenías et al., 1996; Femenías \& Sans, 2000; Moreno et al., 2014). Además, son muy abundantes los restos líticos, animales, vegetales y cerámicos (Curbelo et al., 1990; Oliveiro \& Campos, 2001).

Fechados radiocarbónicos sitúan el período de ocupación entre el $2090 \pm 90$ (KR139, carbón) y el $220 \pm 50$ (URU0014, óseo humano) (Bracco, 2006).

La estratigrafía del montículo se compone de 4 capas de origen antrópico (capas A-D), que se desarrollan por encima del suelo natural (capas E-I). La capa E constituye el primer horizonte del suelo natural enterrado y la capa D la primera ocupación del montículo, y ambas se analizan como resultado de la misma ocupación. Los estudios sedimentológicos (físico-químicos) confirmaron la identidad de las capas antrópicas reconocidas en campo. Los sedimentos que componen el cerrito están enriquecidos en el orden de 10 a 100 veces en sus tenores de materia orgánica, fósforo y potasio en relación a los sedimentos naturales. Presentan valores de $\mathrm{pH}$ más altos, que varían entre las capas y que aumentan con la profundidad desde 6,1 (capa A) hasta 7,3 (capa D). Las variaciones texturales dentro del perfil antrópico no son atribuibles a la lixiviación mecánica y además contiene porcentajes más altos de gravas que los suelos naturales (Durán, 1989). Inicialmente, estas unidades estratigráficas fueron interpretadas como ocupaciones discretas en una dinámica de formación por etapas acotadas y separadas en el tiempo (López Mazz, 1992). El estudio tafonómico del conjunto zooarqueológico de la excavación IA no presenta elementos que permitan inferir la acción significativa de agentes modificadores o removilizadores de los materiales, que además parecen haber sufrido un rápido enterramiento (Moreno, 2017). El material óseo no muestra rodamiento de bordes ni evidencias de pisoteo, e incluso se recuperó un tarso de Blastoceros dichotomus articulado y unido por concreciones carbonáticas (Moreno, 2017: 473).

Únicamente 149 restos $(0,82 \%)$ distribuidos en las capas A-F muestran modificaciones por meteorización. El 97,4\% de éstos se encuentra en el estadio 0 y el restante $2,6 \%$ en el estadio 2 de Behrensmeyer. Taxonómicamente corresponden 
a cérvidos y mamíferos grandes. Los restos de ave, incluida el ñandú, no presentan evidencias de meteorización. Aunque no hay información publicada sobre la presencia de cuevas o galerías, la baja frecuencia de marcas de carnívoros y roedores sumadas $(<1 \%)$ indican que los restos no fueron intensamente atacados por estos animales. Las raíces, por su parte, se detectaron en menos del $1 \%$ del material. La modificación más abundante es la precipitación carbonática, presente en el $25 \%$ del material. Esta modificación aumenta dentro del cerrito a medida que nos acercamos al horizonte arcilloso, pasando de una frecuencia de $0,25 \%$ en la capa A, a más del $49 \%$ en las capas D y E (Moreno, 2017).

El conjunto de los restos de fauna recuperado es muy abundante en comparación con sitios regionales similares, y muestra un excepcional estado de conservación (Moreno, 2017).

Los restos óseos de ñandú se determinaron taxonómica y anatómicamente utilizando la colección de referencia del Museo Nacional de Historia Natural de Montevideo. Las unidades de cuantificación utilizadas fueron el NISP (Number of Identified SPecimens, Número de Restos Identificados) y el MNI (Minimun Number of Individuals, Número Mínimo de Individuos) (Lyman, 2008). Este último se estimó a partir de los elementos anatómicos recuperados.

Los restos se observaron a ojo desnudo y a bajos aumentos (4-10X) para identificar modificaciones de superficie antrópicas y naturales. Se relevaron marcas de carnicería, alteración térmica, fracturación y modificaciones por meteorización, animales, raíces y procesos diagenéticos (Behrensmeyer, 1978; Binford, 1981; Politis \& Madrid, 1988; Fisher, 1995; Behrensmeyer et al., 2003; Cruz, 2011).

Los valores de densidad ósea y de utilidad económica utilizados en este análisis fueron los obtenidos para Rhea pennata dado que ambas especies pertenecen a la misma familia y son anatómicamente similares (Cruz \& Elkin, 2003; Giardina,
2006). Para evaluar la existencia de problemas de equifinalidad se correlacionaron los $\%$ de supervivencia de los elementos recuperados con sus respectivos valores de densidad ósea.

\section{RESULTADOS}

Los restos zooarqueológicos recuperados y analizados en la excavación IA suman 18683, y el $98 \%$ de los mismos se distribuyen entre las capas A-D+E. El 0,1\% del conjunto total (NISP=20) fue determinado como perteneciente a ñandú. Los restos se distribuyen en forma continua y variable desde la capa A hasta la E, con una concentración de 10 restos en la capa D+E (Tabla 1). Anatómicamente corresponden a elementos de las extremidades posteriores: falanges (NISP $=13)$, tarsometatarso distal $(\mathrm{NISP}=6)$ y tibiotarso distal $(\mathrm{NISP}=1)$ (Tabla 1) (Figura 2a y 2b).

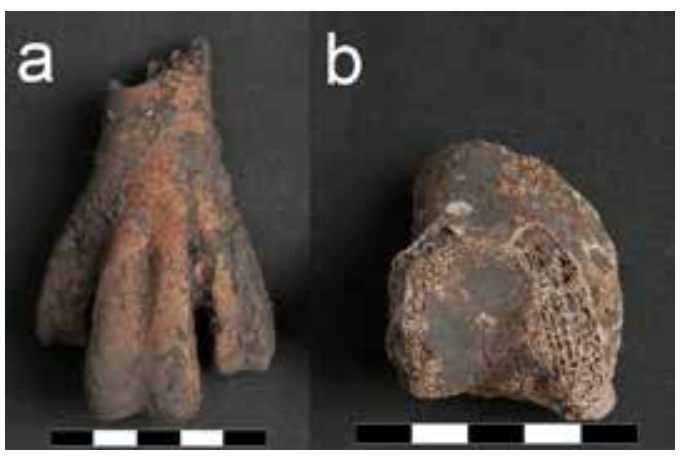

FIGURA 2

Porciones distales de tarsometatarso (a) y tibiotarso (b).

En la Capa A, los 4 restos identificados corresponden a falanges: 2 falanges proximales correspondientes al dedo III (central) y 2 falanges mediales correspondientes al dedo II (lateral). En la capa $\mathrm{B}, \operatorname{los} 5$ restos óseos corresponden a falanges

\begin{tabular}{|c|c|c|c|c|c|c|}
\hline \multirow{2}{*}{ Capa } & \multirow{2}{*}{ NISP } & \multirow{2}{*}{$\%$ NISP } & \multicolumn{4}{|c|}{ Elemento (NISP) } \\
\cline { 4 - 8 } & & & Tibiotarso (distal) & Tarsometatarso (distal) & Falange prox. & Falange med. \\
\hline A & 4 & 20 & & & 2 & 2 \\
\hline B & 5 & 25 & & & 5 & 1 \\
\hline C & 1 & 5 & & & & \\
\hline D+E & 10 & 50 & 1 & 6 & 3 & 3 \\
\hline Total & 20 & 100 & 1 & 6 & 10 & 5 \\
\hline
\end{tabular}

TABLA 1

NISP y \%NISP de los restos de ñandú discriminados por capa; NISP de los elementos anatómicos identificados y NMI. 
proximales: 1 dedo III, 1 dedo IV de pata izquierda, 1 dedo II de pata derecha y 2 restos correspondientes a falanges proximales sin determinar a qué dedo pertenecen. En la capa C, el único resto óseo es una falange medial de dedo no determinado. En la capa $\mathrm{D}+\mathrm{E}$, de los 10 restos óseos, 3 son falanges proximales ( 1 falange proximal de dedo II, 1 proximal de dedo III y 1 proximal de dedo IV), 6 son fragmentos distales de tarsometatarso (4 izquierdos, 1 derecho, y el restante juvenil de lateralidad no determinada) y el restante es un fragmento distal de tibiotarso. En total, para todo el montículo el ñandú alcanza un $\mathrm{NMI}=5$ que surge de los tarsometatarsos (4 izquierdos adultos más 1 juvenil).

\section{MODIFICACIONES ANTRÓPICAS}

El 50\% $(n=10)$ de los restos presentan evidencias de procesamiento antrópico: marcas de corte, alteración térmica y fracturación en estado fresco. Las marcas de carnicería, presentes en tres falanges proximales, corresponden en todos los casos a grupos de cortes. En dos casos las marcas se ubican en la porción medial de la falange, y en un caso en la epífisis distal. Una de las falanges con trazas de

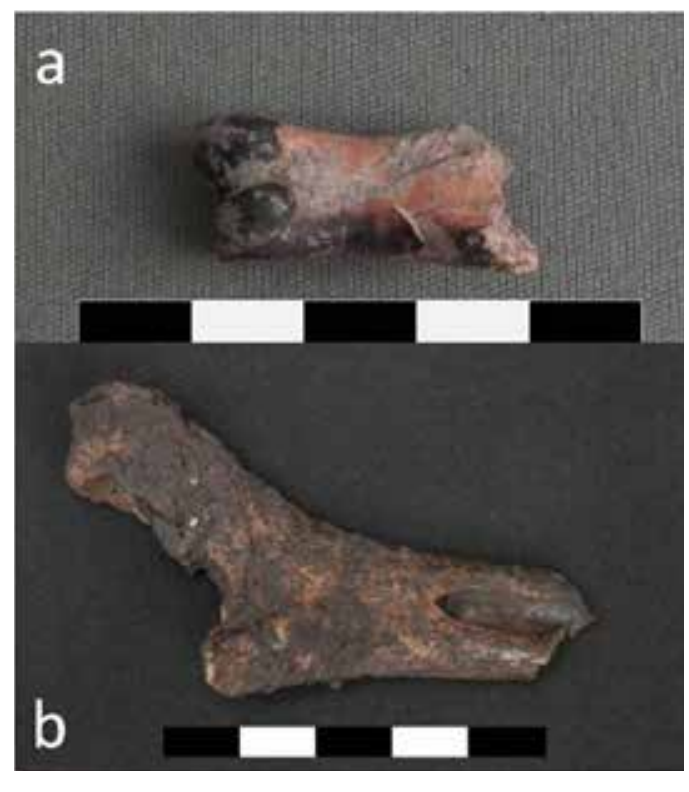

FIGURA 3

Falange proximal quemada (a) y fragmento de instrumento sobre porción distal de tarsometatarso (b). En ambos casos los restos están parcialmente recubiertos de carbonato de calcio. corte, está también parcialmente quemada (Figura 3a) y es el único resto con este tipo de alteración.

La fracturación intencional sobre hueso fresco se reconoció en 7 especímenes: 2 falanges proximales, 4 tarsometatarso distales y 1 tibiotarso distal.

Además un tarsometatarso es un fragmento de instrumento con la superficie fuertemente pulida (Figura 3b).

\section{MODIFICACIONES NATURALES}

Los elementos anatómicos de ñandú se comportan, en relación con la meteorización, como huesos de ungulados (Cruz, 2015), mientras que los elementos de las extremidades anteriores, el cráneo y el esternón sufren una rápida destrucción (Cruz, 2011). En este conjunto, no se detectaron evidencias de meteorización en los restos de ñandú ni modificaciones de origen animal o vegetal.

El 50\% de los restos de ñandú están completos (falanges), mientras que el 15\% está fracturado naturalmente.

La modificación natural más frecuente es la precipitación carbonática sobre la superficie de los restos, presente en 11 restos (55\%) (Figura 3 a y b). Este tipo de concreción puede dificultar la visualización de las superficies, ocultando modificaciones como trazas corte y alteraciones por meteorización o por acción de animales y vegetales, entre otras.

\section{DISCUSIÓN Y CONCLUSIONES}

La baja presencia de rheidos y la sobrerepresentación de elementos de las extremidades traseras es una constante tanto de los registros zooarqueológicos del sudeste uruguayo, como de los de Pampa y Patagonia argentinas (Belardi, 1999; Fernández, 2000; Cruz y Elkin, 2003; Acosta \& Loponte, 2010; Frontini \& Picasso, 2010, entre otros). Las causas esgrimidas para explicar esta escasez son variadas. Tienen que ver con factores culturales (costo de obtención o estrategias de traslado y consumo), metodológicos (problema de identificación taxonómica), ecológicos (baja densidad de estas aves en el pasado) y tafonómicos (preservación diferencial de partes anatómicas asociada a la densidad mineral 
ósea) (Fernández, 2000; Cruz, 2005; Fernández et al., 2001).

El conjunto que analizamos aquí se asemeja a los conjuntos recuperados en Pampa y Patagonia en la medida en que los restos son escasos y están representadas, en este caso únicamente, las extremidades posteriores. Los conjuntos zooarqueológicos del este de Uruguay se caracterizan por una fragmentación muy alta, que ronda el $90 \%$ (Pintos, 2000; Iriarte, 2003; Moreno, 2017). En el caso que presentamos aquí, los restos fragmentados representan el 94\% del total del conjunto zooarqueológico (Moreno, 2017). De acuerdo a lo propuesto por Fernández (2000) la alta fragmentación puede explicar en parte la escasez al contribuir a la pérdida diagnóstica ya que los fragmentos de diáfisis de los huesos largos de ñandú pueden confundirse por su grosor con los de mamíferos de tamaño mediano a grande (Cruz \& Elkin, 2003). La densidad poblacional del ñandú en la época de ocupación del sitio no se conoce, pero en la actualidad es un animal con una amplia distribución y que mantiene poblaciones importantes (Aspiroz et al., 2012).

La tendencia anatómica, por su parte, podría estar relacionada con aspectos culturales, naturales o una combinación de ambos:

1. Transporte, procesamiento y consumo selectivos relacionados con la anatomía económica (Fernández et al., 2001; Cruz, 2005; Giardina, 2006).

2. Preservación diferencial relacionada con las diferencias en la densidad ósea dentro del esqueleto (Fernández et al., 2001; Cruz \& Elkin, 2003; Cruz, 2005) y

3. Destrucción diferencial por carnívoros, que también va a estar condicionada por la densidad ósea diferencial (Belardi, 1999; Fernández, 2000).

El sesgo en la representación anatómica no sería una consecuencia de la acción de carnívoros en este caso, ya que ni el conjunto de restos de Rhea americana, ni el conjunto general permite inferir la acción de estos animales de forma significativa sobre el conjunto (Moreno, 2017).

En relación con la densidad mineral ósea, el conjunto está compuesto por elementos de valores medios y medio-bajos, como el tarsometatarso y tibiotarso distales, y de valores altos como las falanges proximales y mediales (Tabla 2). No se tomó en cuenta el fragmento de tarsometatarso correspondiente a un juvenil dado que la densidad mineral ósea varía con la edad (Álvarez et al., 2010). No se identificaron elementos de mayor densidad relativa como las diáfisis de los huesos largos posteriores, el húmero, la pelvis, la mandíbula e incluso las vértebras torácicas y cervicales (Cruz \& Elkin, 2003). En el caso de los fragmentos de diáfisis, como ya se comentó más arriba, su ausencia podría estar relacionada con la alta fragmentación del conjunto general.

Para investigar la existencia de un problema de equifinalidad en la muestra se aplicó un test de correlación entre el \% de supervivencia (\%SUP) de cada elemento anatómico y la densidad ósea correspondiente (Lyman, 1994) no encontrándose una asociación estadísticamente significativa entre estas dos variables $\left(r_{s}=-0,10541 ; p=1\right)$ (Figura 4).

La falta de correlación estadística seguramente está influenciada por el tamaño de la muestra, por lo que los resultados no pueden interpretarse como evidencia directa de que el conjunto es el resultado únicamente de actividades humanas. Esta falta de correlación indica únicamente que la densidad mineral ósea no es un factor significativo en la conformación anatómica de este conjunto. De cualquier manera, y teniendo en cuenta que existen otros procesos que también afectan diferencialmente a un mismo conjunto zooarqueológico, un estudio más completo en torno a la densidad ósea debería llevarse a cabo, incluyendo el comportamiento de las otras especies recuperadas para testear si el conjunto general presenta problemas de equifinalidad.

Un factor más a considerar es el transporte diferencial de partes anatómicas y los Índices de Utilidad Económica. De acuerdo a los modelos de

\begin{tabular}{|l|c|c|c|c|c|}
\hline Elemento & NME & MAU & \%MAU & \%SUP & Densidad ósea (Cruz \& Elkin, 2003 \\
\hline Tibiotarso distal & 1 & 0,5 & 20 & 12,5 & 0,44 \\
\hline Tarsometatarso distal & 5 & 2,5 & 100 & 75 & 0,54 \\
\hline Falange post. 1 & 10 & 1,7 & 68 & 41,7 & 0,88 \\
\hline Falange post. 2 & 3 & 0,5 & 20 & 12,5 & 1,2 \\
\hline
\end{tabular}

TABLA 2

MAU, \%MAU, \%SUP y valores de densidad ósea correspondientes para los 4 individuos adultos. 


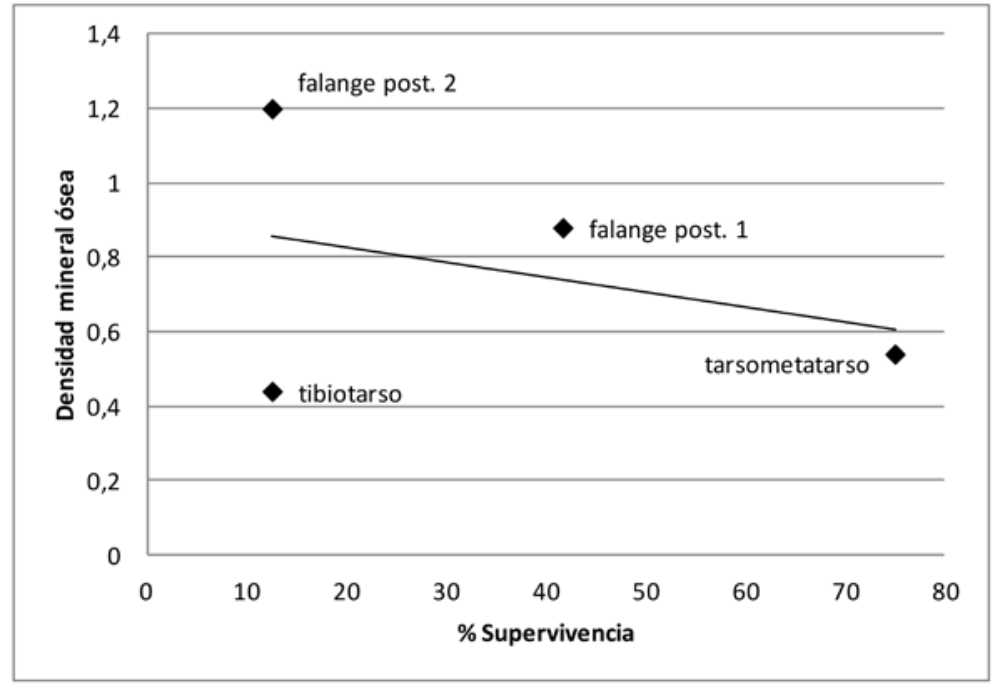

FIGURA 4

Gráfico de correlación entre el \% de supervivencia de cada parte anatómica y su respectiva densidad ósea.

expectativas arqueológicas propuestos por Giardina (2006) (ver más arriba) el conjunto estudiado no se corresponde con un sitio de captura ya que no se recuperaron vértebras cervicales ni miembro anterior. También difiere de la expectativa para un sitio de consumo, ya que están ausentes la cintura pélvica y el fémur, aunque fragmentos de diáfisis de este último, que poseen la misma densidad ósea que las falanges posteriores mediales, pueden estar mezclados con el material no determinado. La pelvis, por su parte, posee una densidad ósea intermedia, es más densa que las epífisis distales de tibiotarso y tarsometatarso, pero menos densa que las falanges posteriores mediales (Cruz \& Elkin, 2003), por lo que no sería afectada por una preservación diferencial vinculada a la densidad. El aprovechamiento de grasa, por otra parte, quedaría evidenciado por la presencia de esternón, cintura pélvica y escápula-coracoides. Tanto el esternón, como la escápula y el coracoides poseen valores de densidad ósea que están en el entorno de los valores del tarsometatarso distal, por lo que en este caso tampoco se trataría de un problema de preservación diferencial vinculado a la densidad (Cruz \& Elkin, 2003). La presencia de miembros posteriores, incluyendo falanges, está relacionada, según Giardina (2006), con el aprovechamiento de la médula, la piel y los tendones, a los que se puede agregar los huesos largos como materias primas. Uno de los tarsometatarsos recuperados es, de hecho, un fragmento de instrumento, por lo que la utilización del ñandú como fuente de materias primas duras en este sitio está demostrada. Parte de los huesos largos y las falanges muestran evidencias de fracturación intencional que pueden asociarse al aprovechamiento de la médula.

Teniendo todo esto en cuenta, podría descartarse que se trate de un sitio de captura o de consumo de grasa, ya que la representatividad anatómica es más acorde con un sitio de consumo de carne, médula y productos no comestibles. De cualquier manera, no debe olvidarse que lo reducido de la muestra no permite descartar del todo problemas de equifinalidad. Los perfiles anatómicos en conjuntos zooarqueológicos no son suficientes en sí mismos para inferir estrategias de subsistencia o determinar funcionalidades. Incluso aunque se combinen con las modificaciones antrópicas y tafonómicas, deben ser acompañados de otras variables que disminuyan los efectos de la acción de procesos naturales o antrópicos que produzcan patrones similares (Cruz \& Elkin, 2003).

Debe destacarse la excepcionalidad del registro de ñandú en este sitio, tanto en cantidad de restos como en número mínimo de individuos. Hasta ahora, el ñandú era considerado un recurso muy minoritario, sino directamente ausente, en la prehistoria de esta región y este caso redimensiona su importancia y sugiere que se trata de un taxón subrepresentado. 


\section{AGRADECIMIENTOS}

Al MSc. Andrés Rinderknecht por el acceso a la colección de referencia del Museo Nacional de Historia Natural y su ayuda en la determinación del material. Agradezco a la evaluadora y los editores del manuscrito cuyos comentarios y sugerencias mejoraron notablemente este trabajo.

\section{REFERENCIAS}

Acosta, A. \& Loponte, D. 2010: Comparando estrategias de explotación faunística en el humedal del Paraná inferior: cazadores-recolectores versus horticultores amazónicos. En: Gutiérrez, M.; De Nigris, M.; Fernández, P.; Giardina, M.; Gil, A.; Izeta, A.; Neme, G. \& Yacobaccio, H. (eds.): Zooarqueología a principios del siglo XXI: Aportes teóricos, metodológicos y casos de estudio: 177-188. Editorial Espinillo, Buenos Aires.

Álvarez, M.C.; González, M.; Massigoge, A. \& Kaufmann, C. 2010: La densidad mineral ósea y la variabilidad ontogénica en guanaco (Lama guanicoe). Implicancias para la construcción de marcos de referencia en Zooarqueología. En: Gutiérrez, M.; De Nigris, M.; Fernández, P.; Giardina, M.; Gil, A.; Izeta, A.; Neme G. \& Yacobaccio, H. (eds.): Zooarqueología a principios del siglo XXI: Aportes teóricos, metodológicos y casos de estudio: 95-106. Editorial Espinillo, Buenos Aires.

Aspiroz, A.; Alfaro, M. \& Jiménez, S. 2012: Lista roja de las aves del Uruguay. Una evaluación del estado de conservación de la avifauna nacional con base en los criterios de la Unión Internacional para la Conservación de la Naturaleza. Dirección Nacional de Medio Ambiente, Montevideo.

Bazzano, G.; Martella, M.; Navarro, J.; Bruera, N. \& Corbella, C. 2002: Uso de hábitat por el ñandu (Rhea americana) en un refugio de vida silvestre: implicancias para la conservación y manejo de la especie. Ornitología Neotropical 13(9): 9-15.

Behrensmeyer, A.K. 1978: Taphonomic and ecologic information from bone weathering. Paleobiology 4(2): 150-162. doi:10.2307/2400283.

Behrensmeyer, A.K.; Stayton, C.T.; Chapman, R.E. \& PARK, A. 2003: Taphonomy and ecology of modern avifaunal remains from Ambosely Park, Kenya. Paleobiology 29: 52-70.

BELARDI, J.B. 1999: Hay choiques en la terraza. Información tafonómica y primeras implicaciones arqueofaunísticas para Patagonia. Arqueología 9: 163-185.
BINFORD, L.R. 1981: Bones. Ancient men and modern myths. Academic Press, San Diego.

Bracco, R. 2006: Montículos de la Cuenca de la Laguna Merín: Tiempo, Espacio y Sociedad. Latin American Antiquity 17(4): 511-540.

Bracco, R.; Del Puerto, L. \& Inda, H. 2008: Prehistoria y Arqueología de la Cuenca de Laguna Merín. En: Loponte, D. \& Acosta, A. (comps.): Entre la tierra y el agua: arqueología de humedales de Sudamérica: 1-60. AINA, Buenos Aires.

Brum, L. 2009: Análisis arqueofaunístico de huevos de ñandu (Rhea americana) en un sitio prehistórico del litoral atlántico uruguayo. En: Bourlot, T.; Bozzuto, D.; Crespo, C.; Hecht, A.C. \& Kuperszmit, N. (eds.): Entre pasados y presentes II: estudios contemporáneos en ciencias antropológicas: 323-339. AINA- Fundación de Historia Natural Félix de Azara, Buenos Aires.

CABrera, L. 2005: Patrimonio y arqueología en el sur de Brasil y región este de Uruguay: los cerritos de indios. Saldvie 5: 221-254.

Capdepont, I. \& Pintos, S. 2006: Manejo y aprovechamiento del medio por parte de los grupos constructores de montículos: cuenca de la Laguna de Castillos, Rocha - Uruguay. Relaciones de la Sociedad Argentina de Antropología XXXI: 117-132.

CRuz, I. 2005: La densidad mineral ósea. Importancia metodológica. Investigación y Ciencia 351: 35-37.

- 2011: Tafonomía de huesos de aves. Estado de la cuestión y perspectivas desde el sur del Neotrópico. Antípoda 13: 147-174.

- 2015: Las investigaciones sobre preservación de huesos de aves y mamíferos grandes en Patagonia (Argentina). Archaeofauna 24: 209-224.

Cruz, I. \& Elkin, D. 2003: Structural bone density of the Lesser Rhea (Pterocnemia pennata) (Aves: Rheidae). Taphonomic and archaeological implications. Journal of Archaeological Science 30: 37-44. doi:10.1006/ jasc. 2001.0826

Curbelo, C.; Cabrera, L.; Fusco, N.; Martínez, E.; Bracco, R.; Femenías, J. \& López Mazz, J.M. 1990: Sitio Ch2D01, Area de San Miguel, Depto. de Rocha, R.O. del Uruguay. Estructura de sitio y zonas de actividad. Revista do CEPA 17(20): 333-344.

DuRÁn, A. 1989: Observaciones sobre los suelos del sitio arqueológico Ch2D01. Informe inédito.

Femenías, J. \& Sans, M. 2000: Subsistencia, movilidad y organización social en el sitio monticular CH2D01IA (Rocha, Uruguay): inferencias a partir de las pautas de enterramientos y los restos esqueletarios. En: Durán, A. \& Bracco, R. (eds.): Arqueología de las Tierras Bajas: 383-394. Comisión Nacional de Arqueología, Montevideo. 
Femenías, J.; SAns, M. \& Portas, M. 1996: Enterramientos humanos en el montículo CH2D01, Departamento de Rocha, Uruguay. Coleçao Arqueología 1: 503-518.

FERnÁNDEZ, P.M. 2000: Rendido a tus pies: acerca de la composición anatómica de los conjuntos arqueofaunísticos con restos de Rheiformes de Pampa y Patagonia. En: Belardi, J.B.; Carballo, F. \& Espinosa, S. (eds.): Desde el país de los gigantes. Perspectivas arqueológicas en Patagonia. Tomo II: 573-586. Universidad Nacional de la Patagonia Austral, Río Gallegos.

Fernández, P.; Cruz, I. \& Elkin, D. 2001: Densidad mineral ósea de Pterocnemia pennata (Aves: Rheidae). Una herramienta para evaluar frecuencias anatómicas en sitios arqueológicos. Relaciones de la Sociedad Argentina de Antropología XXVI: 243-260.

FISHER, J.W. 1995: Bone surface modifications in zooarchaeology. Journal of Archaeological Method and Theory 2(1): 7-68. doi:10.1007/BF02228434

Frontini, R. 2013: Aprovechamiento faunístico en entornos acuáticos del sudoeste bonaerense durante el Holoceno (6900-700 años AP). Relaciones de la Sociedad Argentina de Antropología XXXVIII(2): 493-519.

Frontini, R. \& Picasso, M. 2010: Aprovechamiento de Rhea americana en la localidad arqueológica El Guanaco. En: Gutiérrez, M.; De Nigris, M.; Fernández, P.; Giardina, M.; Gil, A.; Izeta, A.; Neme, G. \& Yacobaccio, H. (eds.): Zooarqueología a principios del siglo XXI: Aportes teóricos, metodológicos y casos de estudio: 1-11. Editorial Espinillo, Buenos Aires.

Gianotti, C. 2005: Arqueología del paisaje en Uruguay. Origen y desarrollo de la arquitectura en tierra y su relación con la construcción del espacio doméstico en la prehistoria de las Tierras Bajas. En: Mameli, L. \& Muntañola, E. (eds.): América Latina, realidades diversas. Aula Oberta 2001-2005: 104-123. Casa América-Catalunya, Barcelona.

GiardinA, M.A. 2006: Anatomía económica de Rheidae. Intersecciones en Antropología 7: 263-276.

InAC, 2003: Manual de cortes de ñandu (Rhea americana) y subproductos. INAC, Montevideo.

IRIARTE, J. 2003: Mid-Holocene emergent complexity and landscape transformation: the social construction of early formative communities in Uruguay, La Plata Basin. PhD Dissertartion, University of Kentucky.

IRIARTE, J. 2006: Landscape transformation, mounded villages and adopted cultigens: the rise of Early Formative communities in South-Eastern Uruguay. World Archaeology 38(4): 644-663.

López MAzz, J.M. 1992: Aproximación a la génesis y desarrollo de los cerritos de la zona de San Miguel
(Rocha). En: Ediciones del Quinto Centenario Tomo 1: 77-96. Facultad de Humanidades y Ciencias de la Educación, Montevideo.

- 2001: Las estructuras tumulares (cerritos) del litoral atlántico uruguayo. Latin American Antiquity 12(3): 231-255.

Lyman, R.L. 1994: Vertebrate Taphonomy. Cambridge University Press, Cambridge.

- 2008: Quantitative Paleozoology. Cambridge University Press, Cambridge.

Moreno, F. 2016: La gestión animal en la prehistoria del este de Uruguay: de la economía de amplio espectro al control de animales salvajes. Tessituras 4: 161-187.

- 2017: Modificaciones naturales y antrópicas en el conjunto zooarqueológico del sitio Ch2D01, excavación IA (sudeste uruguayo): aportes a la discusión de los procesos de formación. Cuadernos do LEPAARQ XIV: 458-479.

Moreno, F.; Figueiro, G. \& Sans, M. 2014: Huesos mezclados: restos humanos de subadultos en el conjunto arqueofaunístico de un sitio prehistórico en el Este de Uruguay. Revista Argentina de Antropología Biológica 16(2): 65-78.

Moreno, F.; Figueiro, G.; Mannise, N.; Iriarte, A.; González, S.; Mauricio, J. \& Cosse, M. 2016: Use of next-generation molecular tools in archaeological neotropical deer sample analysis. Journal of Archaeological Science: Reports 10: 403-410. doi:10.1016/j. jasrep.2016.11.006

Oliveiro, J. \& CAmpos, S. 2001: Análisis de partículas biosilíceas en la matriz del sitio arqueológico Ch2D01. En: Arqueología uruguaya hacia el fin del Milenio. IX Congreso Nacional de Arqueología. Tomo 1: 539550. Gráficos del Sur, Montevideo.

Pintos, S. 1995: Manejo prehistórico de recursos faunísticos en los humedales del Este. Tesis de grado. Universidad de la República, Montevideo.

- 2000: Economía "húmeda" del este de Uruguay: el manejo de recursos faunísticos. En: Durán, A. \& Bracco, R. (eds.): Arqueología de las Tierras Bajas: 249-270. Comisión Nacional de Arqueología, Montevideo.

Pintos, S. \& GianotTi, C. 1995: Arqueofauna de los Constructores de Cerritos: "Quebra" y Requiebra. En: Consens, M.; López, J.M. \& Curbelo, C. (eds.): Arqueología en el Uruguay. VIII Congreso Nacional de Arqueología Uruguaya: 79-91. Editorial Surcos, Montevideo.

Politis, G. \& MAdRID, P. 1988: Un hueso duro de roer: análisis preliminar de la tafonomía del Sitio Laguna Tres Reyes 1 (Pdo. de Adolfo González Chávez, Pcia. de Buenos Aires). En: Ratto, N. \& Haber, A. (eds.): 
De procesos, contextos y otros huesos: 29-44. Universidad de Buenos Aires, Buenos Aires.

Probides, 1999: Plan Director. Reserva de biósfera Bañados del Este. Uruguay, Rocha. PROBIDES, Uruguay.
RebOREDA, J.c. \& FernándeZ, G.J. 2005: Estudios sobre ecología del comportamiento del ñandú, Rhea americana. Fundación para la Conservación de las Especies y Medio Ambiente, Buenos Aires. 